\title{
Multidimensional Analysis on the Effect of Vocal Function Exercises on Aged Vocal Fold Atrophy.
}

\section{$\operatorname{AUTHOR}(\mathrm{S}):$}

Kaneko, Mami; Hirano, Shigeru; Tateya, Ichiro; Kishimoto, Yo; Hiwatashi, Nao; Fujiu-Kurachi, Masako; Ito, Juichi

\section{CITATION:}

Kaneko, Mami ... [et al]. Multidimensional Analysis on the Effect of Vocal Function Exercises on Aged Vocal Fold Atrophy.. Journal of voice 2015, 29(5): 638-644

\section{ISSUE DATE:}

2015-09

URL:

http://hdl.handle.net/2433/202589

\section{RIGHT:}

(c) 2015. This manuscript version is made available under the CC-BY-NC-ND 4.0 license

http://creativecommons.org/licenses/by-nc-nd/4.0/; The full-text file will be made open to the public on 1 September 2016 in accordance with publisher's 'Terms and Conditions for Self-Archiving'.; この論文は出版社版でありません。引 用の際には出版社版をご硫認ご利用ください。; This is not the published version. Please cite only the published version. 


\title{
Multi-dimensional Analysis on the Effect of Vocal Function Exercises on Aged Vocal Fold Atrophy \\ (Abbreviation: Vocal Function Exercises for aged atrophy)
}

\author{
Mami Kanekoํㅗ SLP \\ Shigeru Hirano ${ }^{1}, \mathrm{MD}, \mathrm{PhD}$ \\ Ichiro Tateya ${ }^{1}, \mathrm{MD}, \mathrm{PhD}$ \\ Yo Kishimoto ${ }^{1}, \mathrm{MD}, \mathrm{PhD}$ \\ Nao Hiwatashi ${ }^{1}, \mathrm{MD}$ \\ Masako Fujiu-Kurachi², SLP, PhD \\ Juichi Ito ${ }^{1}, \mathrm{MD}, \mathrm{PhD}$
}

${ }^{1}$ Department of Otolaryngology-Head and Neck Surgery, Graduate School of Medicine, Kyoto University, Kyoto.

2 Department of Eating Disorder and Dysphagia, the University of Niigata

Rehabilitation Graduate School, Niigata.

$\checkmark \quad$ This study was supported by the Advanced Research for Medical Products

Mining Program of the National Institute of Biomedical Innovation (NIBIO).

$\checkmark$ Presented at the Voice Foundation's 43rd Annual Symposium, Philadelphia,

Pennsylvania, U.S.A., May 28 - June 1, 2014.

$\checkmark \quad$ No financial disclosure.

$\checkmark$ Conflict of Interest: None 


\section{Correspondence to:}

Shigeru Hirano, MD, PhD. Department of Otolaryngology-Head \& Neck Surgery, Graduate School of Medicine, Kyoto University, Sakyo-ku, Kyoto 606-8507, Japan. Tel: +81-75-751-3346, Fax:+81-75-751-7225, E-mail: hirano@ent.kuhp.kyoto-u.ac.jp 


\section{Abstract(<250 words)}

Objectives: Age-related voice change is characterized as weak, harsh, and breathy. These changes are caused by histologic alteration of the lamina propria of the vocal fold mucosa as well as atrophy of the thyroarytenoid muscle. Several therapeutic strategies involving laryngeal framework surgery and injection laryngoplasty have been tried, but effects have been limited. Vocal function exercises (VFE) have been used to treat age-related vocal fold atrophy, although the effectiveness has been shown with limited analysis. The present study aims to determine the effectiveness of VFE for the treatment of aged atrophy using multi-dimensional analysis.

Study design: retrospective

Methods: Sixteen patients with vocal fold atrophy aged 65 to 81 years underwent voice therapy using VFE. Six patients with vocal fold atrophy aged 65 to 85 years were involved as historical control group. GRBAS scale, stroboscopic examinations, aerodynamic assessment, acoustic analysis and Voice Handicap Index-10 (VHI-10) were performed pre and post. Normalized mucosal wave amplitude (NMWA), normalized glottal gap (NGG) and bowing index (BI) were measured by image analysis during stroboscopic examinations.

Results: Post VFE, significant improvements were shown in GRBAS, MPT, jitter, NMWA, NGG and VHI-10 though BI hasn't change significantly. There were no significant 
improvements in the historical control.

Conclusions: The data suggest that VFE produces significant improvement in subjective, objective and patient self-evaluation, and deserves further attention as a treatment for aged atrophy of the vocal fold. It was also suggested that VFE does not improve the vocal fold bowing but may improve muscular function during voicing.

Key Words: aged vocal fold atrophy, voice therapy, vocal function exercises, multi-dimensional analysis 


\section{Introduction}

Voice quality can profoundly impact the quality of life and limit social interaction ${ }^{1}$.

Voice problems are typically non-life threatening but can substantially impact an individual's quality of life ${ }^{2}$. Unfortunately, the voice of elderly people often declines with age $^{3,4}$. The aged voice is characterized as breathy, weak and strained. Common vocal symptoms include decreased loudness, inconsistent hoarseness, decreased pitch for females, increased pitch for males ${ }^{5,6}$, and increased vocal effort and vocal fatigue ${ }^{7,8}$. These changes are caused by histologic alteration of the lamina propria of the vocal fold mucosa as well as atrophy of the thyroarytenoid (TA) muscle ${ }^{9}$. Vocal fold atrophy involves either the muscle, the mucosa, or another structure within the vocal fold ${ }^{10}$. In addition, decreased lung elasticity, vital capacity and respiratory strength might further degrade phonatory efficiency ${ }^{11}$. Therefore, it is likely that age-related systemic and structural changes all contribute to a decline in voice quality and vocal function in the elderly.

Treatments for aged atrophy include surgical approaches such as framework surgery and injection laryngoplasty, but they are invasive and not always indicated or desired ${ }^{12,13}$. Lu et al ${ }^{14}$ reported only modest functional improvements following type I 
thyroplasty in patients with aged atrophy. Hirano et al. ${ }^{15}$ reported that recovery of vibratory function is limited with surgery, because the histologic changes within the mucosa do not improve, thus the quality of voice rarely shows sufficient improvement. Hirano et al. also examined the regenerative effects of basic fibroblast growth factor (bFGF) on aged vocal folds in 10 patients in a clinical trial. The results showed good recovery of vibratory properties, as well as aerodynamic and acoustic function, with no major adverse effects ${ }^{15}$. However, further study with a larger number of patients is warranted.

Regarding voice therapy, several studies have reported the improvement of voice in aged people. Ramig et al. ${ }^{7}$ examined the effects of a 1-month Lee Silverman Voice Treatment on three aged individuals. They reported increases in loudness, sound pressure level and improvements in perceived voice quality. Siracusa et al. ${ }^{16}$ reported immediate short-term benefit to aged voice quality after a semi-occluded vocal tract exercise. Although there were no self-perceived vocal improvements after these exercises, an immediate improvement was reported for perceptual assessment of voice quality and loudness.

Vocal function exercises (VFE), as described by Stemple et al. ${ }^{17}$, were thus designed to strengthen and rebalance the subsystems involved in voice production (i.e., 
respiration, phonation, and resonance) through a program of systematic exercise. VFE represents a series of systematic exercises designed ostensibly to strengthen and rebalance the laryngeal musculature, increase or improve vocal fold adduction, and coordinate the subsystems of voice production ${ }^{17}$. Although the assumptions pertaining to these exercises have not been empirically validated, the exercises have shown to be useful for improving select aspects of vocal performance of speakers with healthy voices $^{17}$, singers ${ }^{18}$ and voice-disordered teachers ${ }^{19}$.

The effectiveness of VFE for aged atrophy has been reported with limited results. Gorman et al. ${ }^{11}$ examined an elderly male cohort diagnosed with presbylaryngis $(n=19)$ who completed a 12 -week course of VFE; they found an improvement in maximum phonation time in addition to improvements in airflow measures, suggesting improved glottal sufficiency. Sauder et al. 20 investigated the effects of VFE as a primary treatment for presbyphonia in nine elderly patients (two women and seven men). After 6 weeks of VFE, patients reported significant reductions in Voice Handicap Index scores, phonatory effort levels, voice severity, and audio-perceptual judgments of breathiness and strain. To further evaluate the effects of VFE on aged vocal folds, the present study sought to perform multi-dimensional assessments of VFE on vocal outcome in aged atrophy, comparing with the historical control of vocal fold atrophy patients. 


\section{Materials and methods}

\section{Subjects}

In this study, 16 patients ( 3 women and 13 men), aged 65 to 81 years old (mean age, 72.9 years old), with vocal fold atrophy were treated by VFE, and the vocal outcome were analyzed retrospectively (VFE group). Six patients ( 1 women and 5 men), aged 65 to 85 years old (mean age, 74.2 years old), who didn't want to receive any therapies and have been just observed were used as a historical control (control group). Physiological problems (i.e. vocal fold bowing) were assessed by stroboscopic examination. Each stroboscopic examination was reviewed by a board certified laryngologist to verify the absence of vocal fold pathology. Diagnosis of atrophy was determined by stroboscopic findings including vocal fold bowing and glottal gap. No patient had a history of neurological disease, respiratory disease or smoking. The subjects presented with stable health conditions during the treatment period. There were no professional singers in the group.

\section{Procedures}

Voice therapy consisted of vocal hygiene and vocal education regarding the physiology and functional problems of the larynx and voice, use of resonant tone for 
optimal vocal postures, and VFE to enhance the strength and balance of the vocal fold mechanism ${ }^{21}$. Approximately 8-week-long sessions of behavioral voice therapy involving VFE were performed. These exercises were selected because they are putatively designed to manage many aspects of voice production, including laryngeal tension, breath support, voice onset, and resonance attributes, which are suitable for the aged voice. In the VFE approach, four specific exercises were practiced at home, two times each per day. All exercises were performed as softly as possible, being combined with a forward placement of the tone (i.e., maximizing midfacial vibratory sensations).In order to confirm the compliance of patients to $\mathrm{VFE}$, the patients were instructed to record their progress and compliance every day, including MPT during /i:/ phonation in the "warm up" exercise. All patients recorded the /i:/ MPTs and its progress, and they were instructed to submit them at each visit.

\section{Assessment}

Vocal outcomes were evaluated pre and post VFE. Regarding historical control group, we defined the first assessments as "pre data", and the other assessments at a few months later as "post data".

GRBAS scale, aerodynamic assessment, acoustic analysis, stroboscopic examinations and VHI-10 were completed. Stroboscopic examination was performed 
using a digital video stroboscopy system with $70^{\circ}$ rigid endoscope, Model 9295 (KayPentax, Lincoln Park, NJ). Aerodynamic assessment, which included maximum phonation time (MPT) and mean flow rate (MFR), and intensity were examined with a phonation analyzer (PA-500; Nagashima Co., Osaka, Japan). Acoustic analyses evaluated jitter, shimmer using a Multi-Dimensional Voice Program (Model 5105; KayPentax).

GRBAS is an anchored perceptual analysis. The grade, roughness, breathiness, asthenia, strain (GRBAS) scale was independently evaluated by two trained raters including a laryngologist and a speech pathologist. This scale was first developed by the Japanese Society of Logopedics and Phoniatrics, and has become popular worldwide ${ }^{22}$. The GRBAS scale is scored from 0 to 3 , in which $0=$ within normal limits, $1=$ slight, $2=$ moderate, and $3=$ severe. The ratings of the five subscales $(G, R, B, A, S)$ were summed and the mean rating-score between two raters was calculated. Inter-rater reliability was evaluated using Spearman's rank correlation coefficient, and the result showed significant correlation, with a correlation coefficient of $r=0.8(\mathrm{P}<0.001)$.

Voice Handicap Index (VHI) is a test battery that has been statistically validated. This instrument, completed prior to and after voice therapy by the patient, permits an understanding of the handicapping nature of the voice disorder as perceived by the 
patient. The 30-item VHI examines self-perceived voice severity as related to functional, physical, and emotional issues ${ }^{21,23,24}$. The VHI-10 is a 10 -question adaption of the original VHI. Rosen et al. used item analysis and clinical consensus results to select the most robust items from the VHI from which to form the VHI-10. They suggested that the VHI-10 is a powerful representation of the VHI that takes less time for the patient to complete without a reduction in validity. Thus, the VHI-10 can replace the VHI as an implement to quantify patients' perception of their voice handicap ${ }^{25}$. In the present study, the VHI-10 was evaluated.

NMWA and NGG were used as parameters of vocal fold vibratory function $26-28$. They were measured by analyzing stroboscopic images during vibration. Vocal fold vibration during phonation of a sustained vowel /i:/ at the patient's normal pitch and loudness was recorded through a $70^{\circ}$ endoscope. Normalized mucosal wave amplitude (NMWA) and normalized glottal gap (NGG) were calculated using Image $\mathrm{J}$ software ${ }^{29}$. To calculate NMWA, the distance (d1) from the midline of the glottis to the free edge of the vocal fold was measured at the anteroposterior middle portion of the vocal fold during the closed phase. The closed phase was determined by the motion of the upper and lower lips of the vocal folds. The same distance (d2) was measured at the maximum open phase. The mucosal wave amplitude was defined by subtracting $\mathrm{d} 1$ from $\mathrm{d} 2$ and 
was normalized by dividing this value by the membranous vocal fold length (L). Therefore, the normalized mucosal wave amplitude $(\mathrm{NMWA})=(\mathrm{d} 2-\mathrm{d} 1) / \mathrm{L} \times 100$ (units) 26-28 (Fig.1(A)). Normalized glottal gap (NGG) was calculated by dividing glottic area (a) at maximum closed phase by the square length of the vocal fold. The formula for $\mathrm{NGG}=\mathrm{a} / \mathrm{L}^{2} \times 100$ (units) $26-28($ Fig.1(B))

The degree of vocal fold bowing was quantified in the stroboscopic images using the bowing index (BI) developed by Omori et al. ${ }^{30}$. The degree of bowing was determined at the vocal fold resting position, as shown in Fig.2. The maximum distance (d) between the edge of the vocal fold and the line connecting the anterior commissure and the tip of the vocal process was measured. BI was defined by dividing the distance (d) by the membranous vocal fold length (L). The formula for $B I=d / L \times 100$ (units). The sum of the BI of both vocal folds was used for analysis.

\section{Statistical Test}

Statistical tests using pre and post voice therapy data were completed for each parameter. Significant differences were reported at the alpha level of 0.05. All reported $\mathrm{P}$ values were two-sided. A p value of less than 0.05 was considered significant. Statistical analysis was performed using a paired t-test for MPT, MFR, intensity, jitter, shimmer, NMWA, NGG and BI. The Wilcoxon signed-rank test was used for VHI-10 and 
GRBAS. 


\section{Results}

Table I shows the mean value with standard deviation of each parameter pre and post voice therapy of VFE and control groups.

Aerodynamic assessment

MPT significantly increased in VFE group $(\mathrm{P}=0.0001)$, but there was no statistically significant change in control group $(\mathrm{P}=0.053)$. MFR didn't statistically improve in both groups $(\mathrm{P}=0.75,0.1$, respectively) (Figure 3$)$.

\section{Intensity}

Intensity didn't statistically improve in both groups ( $\mathrm{P}=0.057,0.32$, respectively) (Figure 3).

Acoustic Analysis

Jitter significantly improved in VFE group $(\mathrm{P}=0.014)$, but there was no statistically significant change in control group $(\mathrm{P}=0.99)$. Shimmer didn't statistically improve in both groups ( $\mathrm{P}=0.1,0.83$, respectively) (Figure 3$)$.

\section{Auditory-Perceptual Ratings}

GRBAS scale significantly improved in VFE group $(\mathrm{P}=0.0001)$, but there was no statistically change in control group $(\mathrm{P}=0.11)$ (Figure 3 ). 


\section{Self-Ratings}

VHI-10 significantly improved in VFE group $(\mathrm{P}=0.0001)$, but there was no statistically change in control group $(\mathrm{P}=0.68)$ (Figure 3$)$.

Stroboscopic examination (NMWA, NGG)

NMWA and NGG significantly improved in VFE group $(p=0.0001,0.019$, respectively). There were no statistically changes in control group ( $\mathrm{P}=0.73,0.6$, respectively) (Figure 3). Figure 4 shows representative stroboscopic images of the vocal fold of a 73-year-old female who underwent VFE. The stroboscopic findings prior to VFE showed bilateral vocal fold atrophy with glottic insufficiency; after VFE, vibratory status improved with complete glottic closure.

\section{Bowing Index}

$\mathrm{BI}$ ranged from 6.02 to 12.78 (mean $9.95 \pm 2.15$ ) pre VFE, and 5.05 to 13.71 (mean $9.14 \pm 2.63)$ post VFE. There was no significant difference between pre and post VFE $(\mathrm{P}=0.14)$ (Figure 5). In control group, pre BI ranged from 5.19 to 12.16 (mean 8.52 \pm 3.33 ), and post BI ranged from 5.23 to 10.01 (mean $7.86 \pm 2.08$ ). There was no significant difference between pre and post in control group $(\mathrm{P}=0.43)$ (Figure 5). 


\section{Discussion}

The present study evaluated the effects of VFE as a primary treatment for aged vocal fold atrophy with multi-dimensional analysis, compared with historical control group. While previous results ${ }^{11,20}$ appeared to support the role of behavioral intervention including VFE as a potentially effective treatment for aged vocal fold atrophy, these studies provided limited data set. The present study was designed to investigate the effectiveness of VFE on vocal function in aged vocal fold atrophy using perceptual ratings (GRBAS), aerodynamic assessment (MPT, MFR), intensity, acoustic analysis (jitter, shimmer), vibratory analysis (NMWA, NGG), VHI-10, and the degree of vocal fold bowing (BI). Although BI didn't change significantly, the present study indicated significant improvements in GRBAS, MPT, jitter, NMWA, NGG and VHI-10 after VFE compared with historical control group.

At the resting position of the vocal fold, the TA muscle is slackened and BI may reflect atrophy of the vocal fold mucosa. This suggests there might be little improvement in the fibrotic and atrophic changes of the vocal fold mucosa with VFE, but these results suggest that VFE can improve vibratory status by improving muscular function. 
The lamina propria consists of three layers: the superficial, intermediate, and deep layers. The innermost layer of the vocal fold is the TA muscle ${ }^{31}$. Functionally, the epithelium and superficial layer of the lamina propria form the "cover," and the TA muscle forms the "body" of the vocal fold ${ }^{9,32}$, while intermediate and deep layers form the vocal ligament. This layered structure contributes to the vibratory properties of the vocal fold, which is called the cover body theory. In this model the TA muscle serves as the body by contraction, and the pliable cover vibrates on the body.

The stretching and contracting exercises in VFE target improvements in strength, endurance, stability, and flexibility of the respiratory and phonatory mechanisms. It is suggested that VFE may improve the contractile function of the atrophic TA muscle in elderly vocal folds by repeated training of sustained phonation in pitch and gliding. As a result, the high elastic constant of the TA muscle may lead to better mucosal vibration, which was implicated in the improvement of NMWA and NGG observed in the present study. Jitter is considered to be related to the periodicity or regularity of vocal fold vibration $^{33}$, and can be improved by stabilizing the vibration through VFE. VFE also helps improve respiratory function in accordance with phonatory function, including respiratory strength, and endurance, which might have contributed to the elongation of MPT observed in this study. 
Our study has limitation. Accurate measurement of the degree of bowing of the vocal fold is quite difficult to obtain because the degree of bowing is affected by different conditions of pitch, loudness and compensatory activity of extrinsic muscles. We attempted to correctly and consistently obtain BI by taking measurements during vocal fold vibration at the patient's normal pitch and loudness, and by using images taken at the identical position of the glottis at resting condition. Also, limitations of the study include the small sample size and retrospective analysis of data. We realize that the historical control in retrospective set-up is weak. A historical study was performed because the assumptions pertaining to VFE has not been empirically validated. However we believe that the present results provided additional information regarding the effectiveness of VFE as a vocal training program to mitigate aspects of aged voice. Further study with a larger number of participants or a prospective randomized controlled trial will be warranted. It will also be important to conduct follow-up evaluations to identify any long-term effects of VFE. 


\section{Conclusion}

This study examined the effects of VFE on aged vocal fold atrophy in sixteen patients, compared with historical control group. In our study, the positive effects of VFE for aged vocal fold atrophy have been shown in subjective, objective and patient self-evaluation, including significant improvement in vocal fold mucosal wave amplitude, glottal sufficiency, jitter, MPT, VHI-10, and GRBAS scale even though the degree of vocal fold bowing hasn't changed. This exercise program is thought to improve respiratory and phonatory coordination, strength, and endurance, resulting in better vibratory status of the vocal fold. Although future study is needed to clarify the physiological mechanisms of voice change after VFE, the data suggest that VFE is a useful tool for the treatment of aged vocal fold atrophy. 


\section{References}

1. Etter NM, Stemple JC, Howell DM. Defining the lived experience of older adults with voice disorders. $J$ Voice. 2013;27:61-67.

2. Verdolini K, Ramig LO. Review: occupational risks for voice problems. Logoped Phoniatr Vocol. 2001;26:37-46.

3. Roy N, Stemple J, Merrill RM, Thomas L. Epidemiology of voice disorders in he elderly: preliminary findings. Laryngoscope. 2007;117:628-633.

4. Mueller PB. The aging voice. Semin Speech Lang. 1997 May;18:159-168.

5. Shipp T, Hollien H. Perception of the aging male voice. $J$ Speech Hear Res. 1969;12:703-710.

6. Hartman DE. The perceptual identity and characteristics of aging in normal male adult speakers. J Commun Disord. 1979;12:53-61.

7. Ramig LO, Gray S, Baker K, et al. The aging voice: a review, treatment data and familial and genetic perspectives. Folia Phoniatr Logop. 2001;53:252-265.

8. Gartner-Schmidt J, Rosen C. Treatment success for age-related vocal fold atrophy. Laryngoscope. 2011;121:585-589.

9. Sato K, Hirano M, Nakashima T. Age-related changes of collagenous fibers in the 
human vocal fold mucosa. Ann Otol Rhinol Laryngol. 2002;111:15-20.

10. Luschei ES. Ramig LO, Baker KL, Smith ME. Discharge characteristics of laryngeal single motor units during phonation in young and older adults and in persons with parkinson disease. J Neurophysiol. 1999;81:2131-2139.

11. Gorman S, Weinrich B, Lee L, Stemple JC. Aerodynamic changes as a result of vocal function exercises in elderly men. The Laryngoscope. 2008; 118 : 1900-1903.

12. Tucker HM. Laryngeal framework surgery in the management of the aged larynx. Ann Otol Rhinol Laryngol. 1988;97:534-536.

13. Slavit DH. Phonosurgey in the elderly: a review. Ear Nose Throat $J$. 1999;78:505-509, 512.

14. Lu FL, Casiano RR, Lundy DS, Xue JW. Vocal evaluation of thyroplasty type I in the treatment of nonparalytic glottic incompetence. Ann Otol Rhinol Laryngol. 1998;107:113-119.

15. Hirano S, Tateya I, Kishimoto Y, Kanemaru S, Ito J. Clinical trial of regeneration of aged vocal folds with growth factor therapy. Laryngoscope. 2012;122:327-331.

16. Siracusa MG, Oliveira G, Madazio G, Behlau M. Efeito imediato do exercicio de sopro sonorizado na voz do idoso. [Immediate effect of sounded blowing exercise in the elderly voice]. J Soc Bras Fonoaudiol. 2011;23:27-31. 
17. Stemple JC, Lee L, D'Amico B, Pickup B. Efficacy of vocal function exercises as a method of improving voice production. $J$ Voice. 1994;8:271-278.

18. Sabol JW, Lee L, Stemple JC. The value of vocal function exercises in the practice regimen of singers. $J$ Voice. 1995;8:927-93.

19. Roy N, Gray SD, Simon M, Dove H, Corbin-Lewis K, Stemple JC. An evaluation of the effects of two treatment approaches for teachers with voice disorders: a prospective randomized clinical trial. $J$ Speech Lang Hear R. 2001;44: 286-296.

20. Sauder C, Roy N, Tanner K, Houtz DR, Smith ME. Vocal function exercises for presbylaryngis: a multidimensional assessment of treatment outcomes. Ann Otol Rhinol Laryngol. 2010;119:460-467.

21. Stemple JC, Glaze L, Klaben B. Survey of Voice Management. In: Clinical Voice Pathology : Theory and Management. $4^{\text {th }}$ ed. San Diego: Plural Publishing; 2010;245-249.

22. Hirano M. Clinical Examination of Voice. New York, NY: Springer, 1981

23. Jacobson B, Johnson A, Grywalski C, Silbergleit A, Jacobson G, Benninger MS, Newman CW. The Voice Handicap Index(VHI):development and validation. Am $J$ Speech-Lang Pathol. 1997;6:66-70.

24. Rosen CA, Murry T, Zinn A, Zullo T, Sonbolian M. Voice handicap index change 
following treatment of voice disorders. $J$ Voice. 2000;14:619-623.

25. Rosen CA, Lee AS, Osborne J, Zullo T, Murry T. Development and validation of the voice handicap index-10. Laryngoscope. 2004;114:1549-1556.

26. Hirano S, Bless DM, Nagai H, Rousseau B, Welham NV, Montequin DW, Ford CN. Growth factor therapy for vocal fold scarring in a canine model. Ann Otol Rhinol Laryngol. 2004;113:777-785.

27. Suehiro A, Hirano S, Kishimoto Y, Rousseau B, Nakamura T, Ito J. Treatment of acute vocal fold scar with local injection of basic fibroblast growth factor: a canine study. Acta Otolaryngol. 2010;130:844-850.

28. Ohno S. Hirano S, Kanemaru S, et al. Implantation of an atelocollagen sponge with autologous bone marrow-derived mesenchymal stromal cells for treatment of vocal fold scarring in a canine model. Ann Otol Rhinol Laryngol. 2011;120:401-408.

29. Abramoff MD, Magelhaes PJ, Ram SJ. Image processing with ImageJ. Biophotonics Int. 2004;11:36-42.).

30. Omori K1, Slavit DH, Matos C, Kojima H, Kacker A, Blaugrund SM. Vocal fold atrophy: quantitative glottic measurement and vocal function. Ann Otol Rhinol Laryngol. 1997;106:544-551.

31. Gray SD. Cellular physiology of the vocal folds. Otolaryngol Clin North Am. 
2000;33:679-698.

32. Hirano M, Kakita Y. Cover-body theory of vocal cord vibration. In: Daniloff RG, ed.

Speech Science. San Diego, CA: College Hill Press; 1985:1-46.

33. Colton RH, Casper JK, Leonard R. Understanding Voice Problems: A Physiological

Perspective for Diagnosis and Treatment. 3rd ed. Philadelphia, PA: Lippincott

Williams \& Wilkins; 2006:498. 


\section{Figure legends}

Table I. Phonatory outcome (mean value \pm standard deviation). ${ }^{*}<0.05,{ }^{*} \mathrm{P}<0.01$.

Figure 1. Image analysis of stroboscopic findings

(A) Measurement of normalized mucosal wave amplitude (NMWA). NMWA $=(\mathrm{d} 2-\mathrm{d} 1) / \mathrm{L}$

$\times 100$ (units). d1: distance from the midline of the glottis to the free edge of the vocal

fold at the closed phase. $\mathrm{d} 2$ : the same distance at maximum open phase. L:

membranous vocal fold length

(B) Measurement of normalized glottal gap (NGG). NGG=a/L ${ }^{2} \times 100$ (units). a: glottal area at maximum closed phase. L: membranous vocal fold length

Figure 2. Measurement of bowing index (BI). BI= $d / L \times 100$ (units). $d$ : distance between the edge of the vocal fold and the line connecting the anterior commissure and the vocal process. L: membranous vocal fold length. 
Figure 3. Phonatory outcomes. Post VFE, significant improvements were shown in maximum phonation time (MPT; $\mathrm{P}=0.0001)$, jitter $(\mathrm{P}=0.014)$, GRBAS scale $(\mathrm{P}=0.0001)$, voice handicap index-10 (VHI-10; $\mathrm{P}=0.0001)$, NMWA $(\mathrm{P}=0.0001)$ and NGG $(\mathrm{P}=0.019)$. There were no statistically significant changes in historical control group.

Figure 4.

(A) Pre VFE images of the vocal fold of a 73-year-old female demonstrating bilateral vocal fold atrophy with glottic insufficiency.

(B) Post VFE stroboscopic findings of the vocal fold of the same 73-year-old female indicating improved vibratory status with complete glottic closure.

Figure 5.

Bowing index (BI). Significant improvement was not shown in post VFE nor control group $(\mathrm{P}=0.14,0.43$, respectively). 


\begin{tabular}{|c|c|c|c|c|c|c|}
\hline Assessment & \multicolumn{3}{|c|}{ VFE group } & \multicolumn{3}{|c|}{ Control group } \\
\hline MPT(s) & $14.19( \pm 5.86)$ & $22.25( \pm 7.86)$ & $* * \mathrm{P}=0.0001$ & $22.17( \pm 9.24)$ & $17.67( \pm 9.23)$ & $\mathrm{P}=0.053$ \\
\hline $\mathrm{MFR}(\mathrm{ml} / \mathrm{sec})$ & $162.31( \pm 43.46)$ & $166.75( \pm 47.87)$ & $\mathrm{P}=0.75$ & $167.67( \pm 54.22)$ & $194( \pm 75.6)$ & $\mathrm{P}=0.1$ \\
\hline jitter $(\%)$ & $1.41( \pm 1)$ & $0.89( \pm 0.72)$ & $* \mathrm{P}=0.014$ & $1.43( \pm 0.58)$ & $1.43( \pm 0.66)$ & $\mathrm{P}=0.99$ \\
\hline shimmer $(\%)$ & $3.8( \pm 2.4)$ & $3.01( \pm 2.22)$ & $P=0.1$ & $3.42( \pm 1.19)$ & $3.3( \pm 0.94)$ & $\mathrm{P}=0.83$ \\
\hline GRBAS & $9.44( \pm 1.74)$ & $6.94( \pm 1.18)$ & $* * \mathrm{P}=0.0001$ & $9.08( \pm 2.5)$ & $9.58( \pm 2.06)$ & $\mathrm{P}=0.11$ \\
\hline BI(units) & $9.95( \pm 2.15)$ & $9.14( \pm 2.63)$ & $P=0.14$ & $8.52( \pm 3.33)$ & $7.86( \pm 2.08)$ & $P=0.43$ \\
\hline
\end{tabular}

Abbreviation: SD, standard deviation 
Figure 1

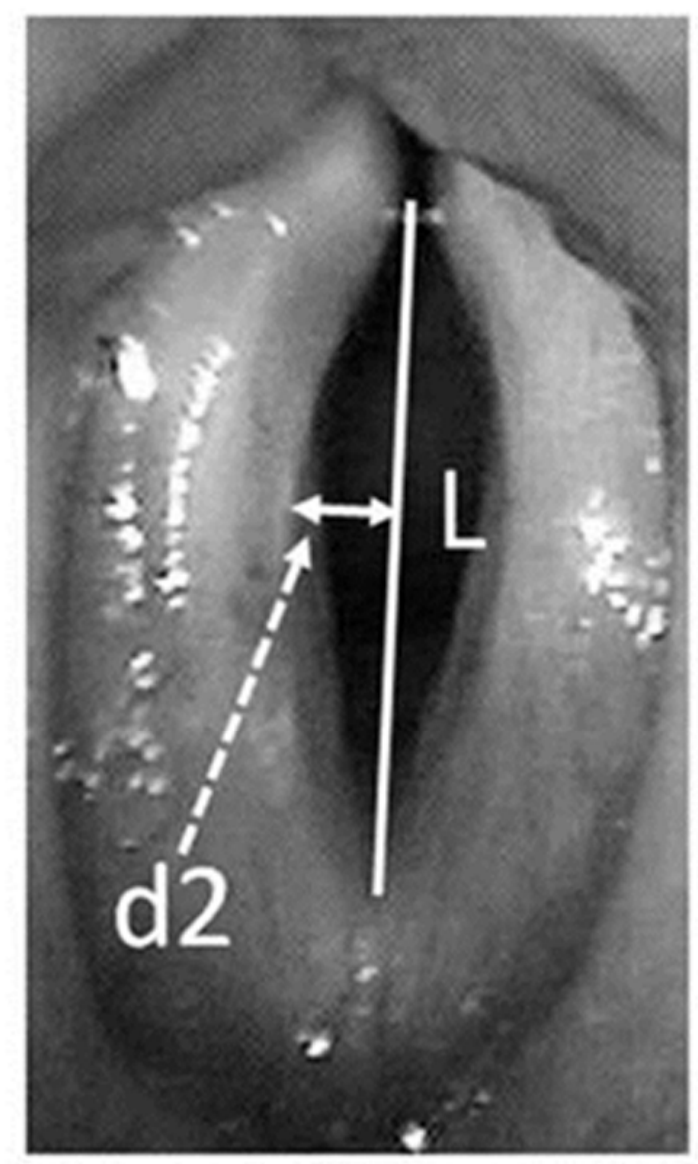

(A)

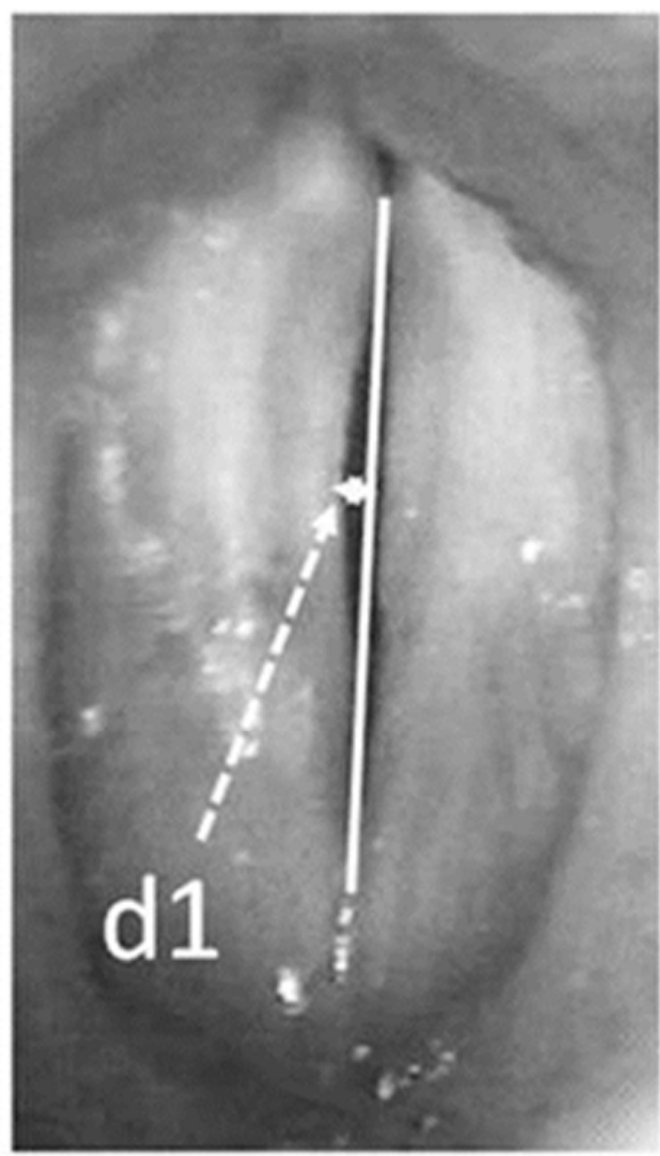

(B)

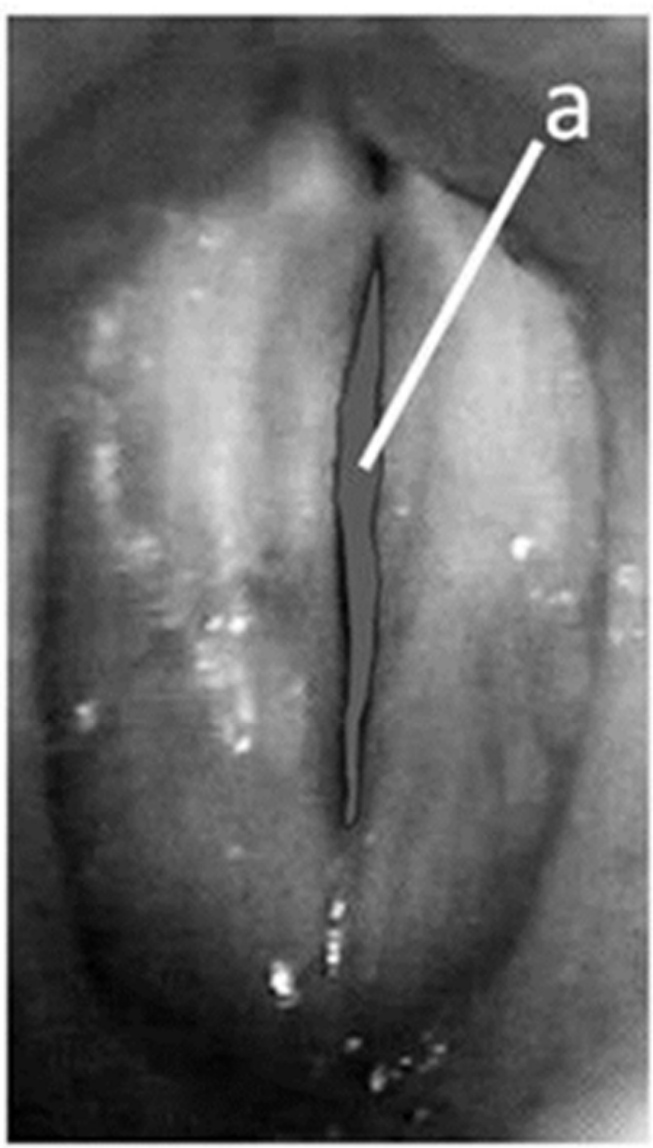


Figure 2

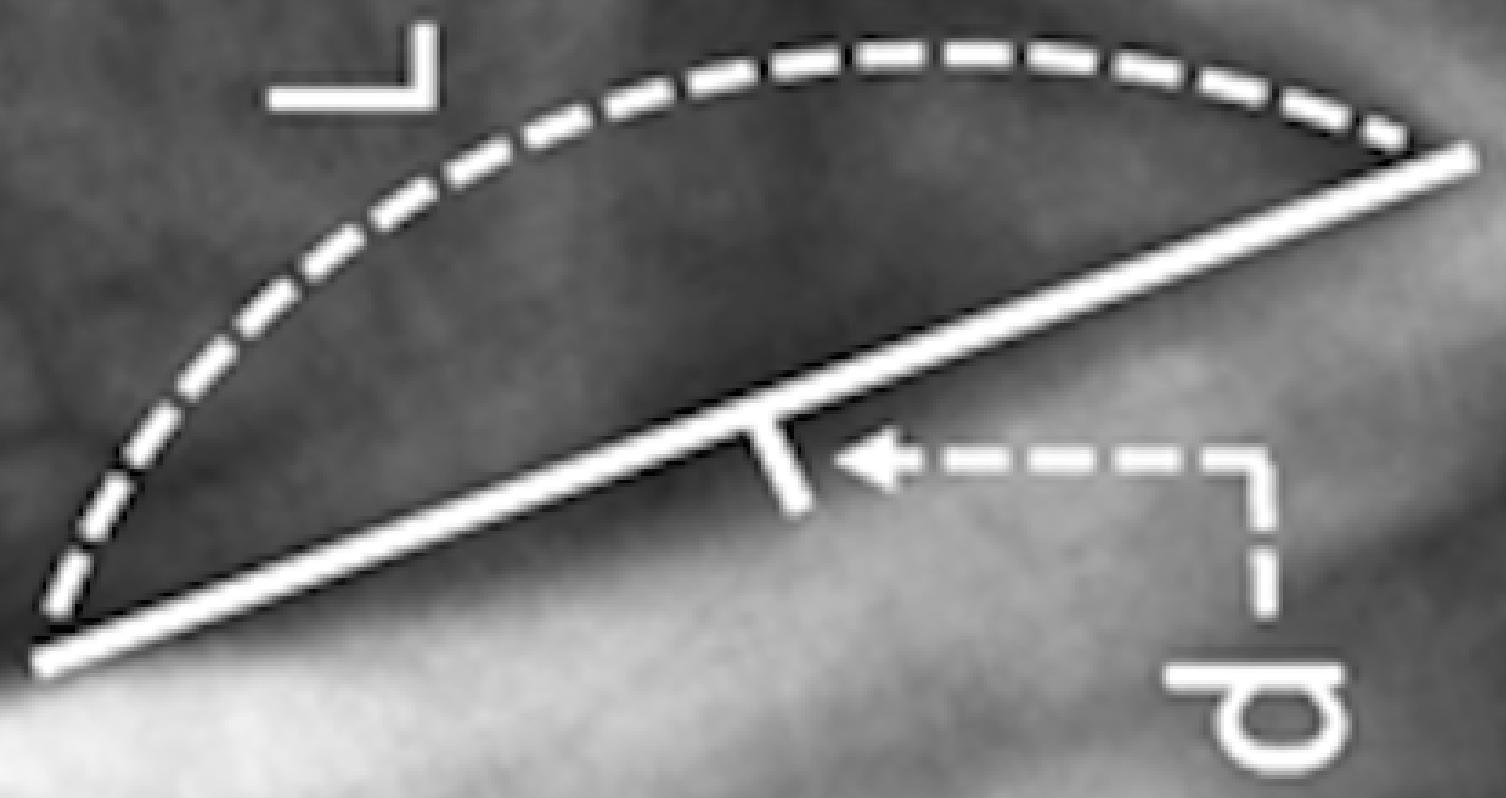



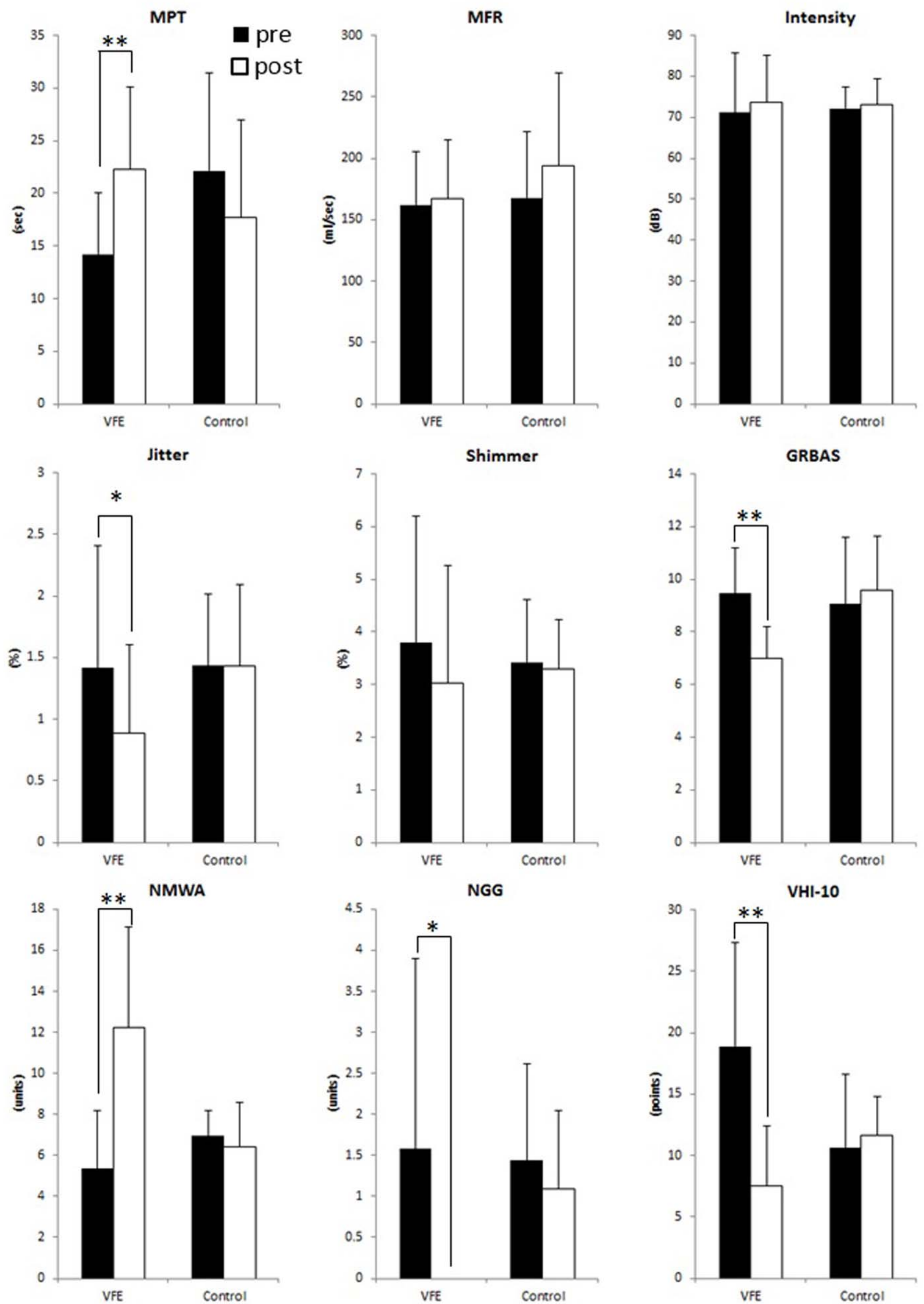
Figure 4

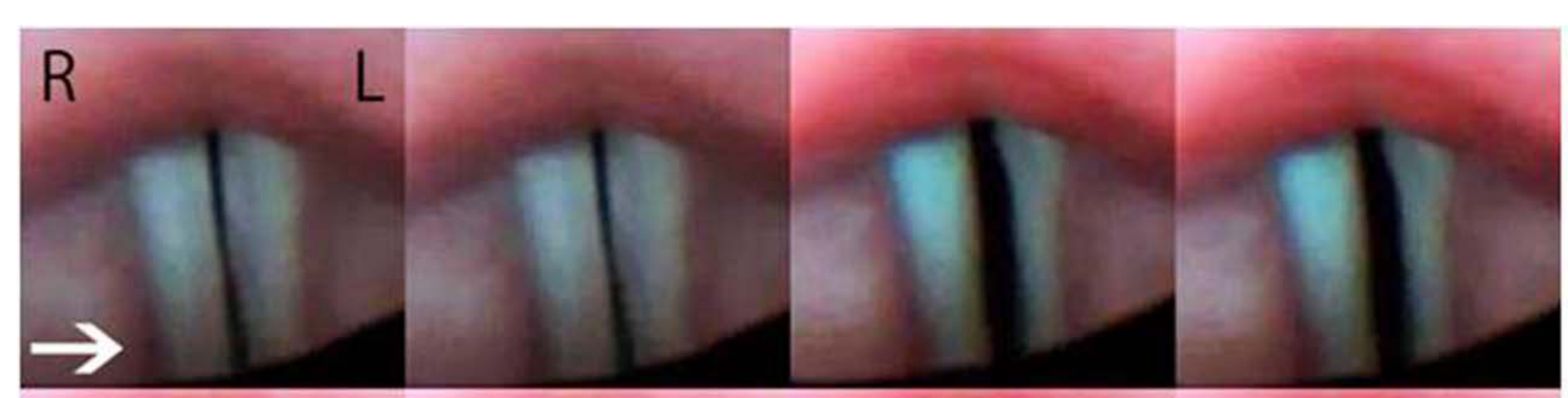

(A)

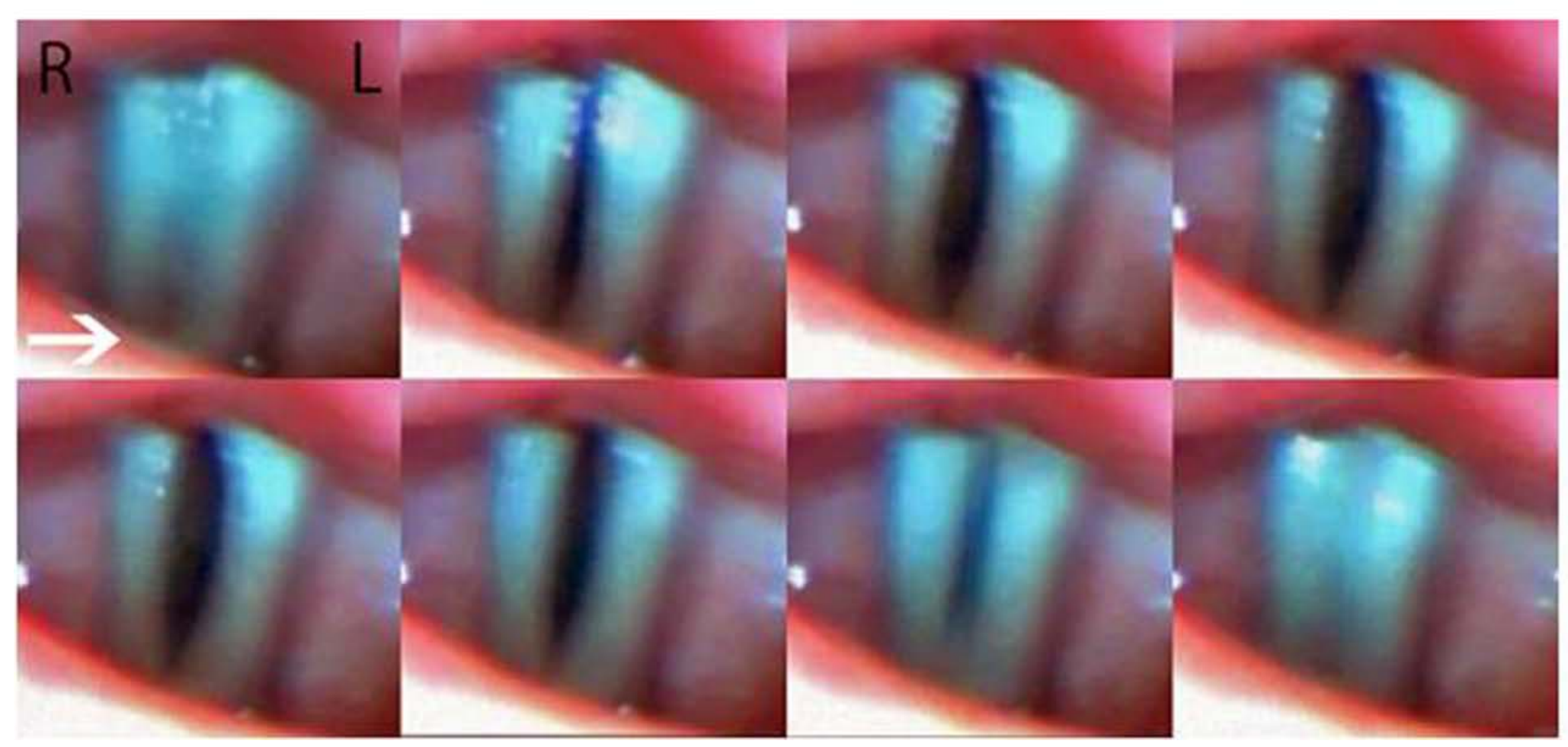

(B) 
Figure 5

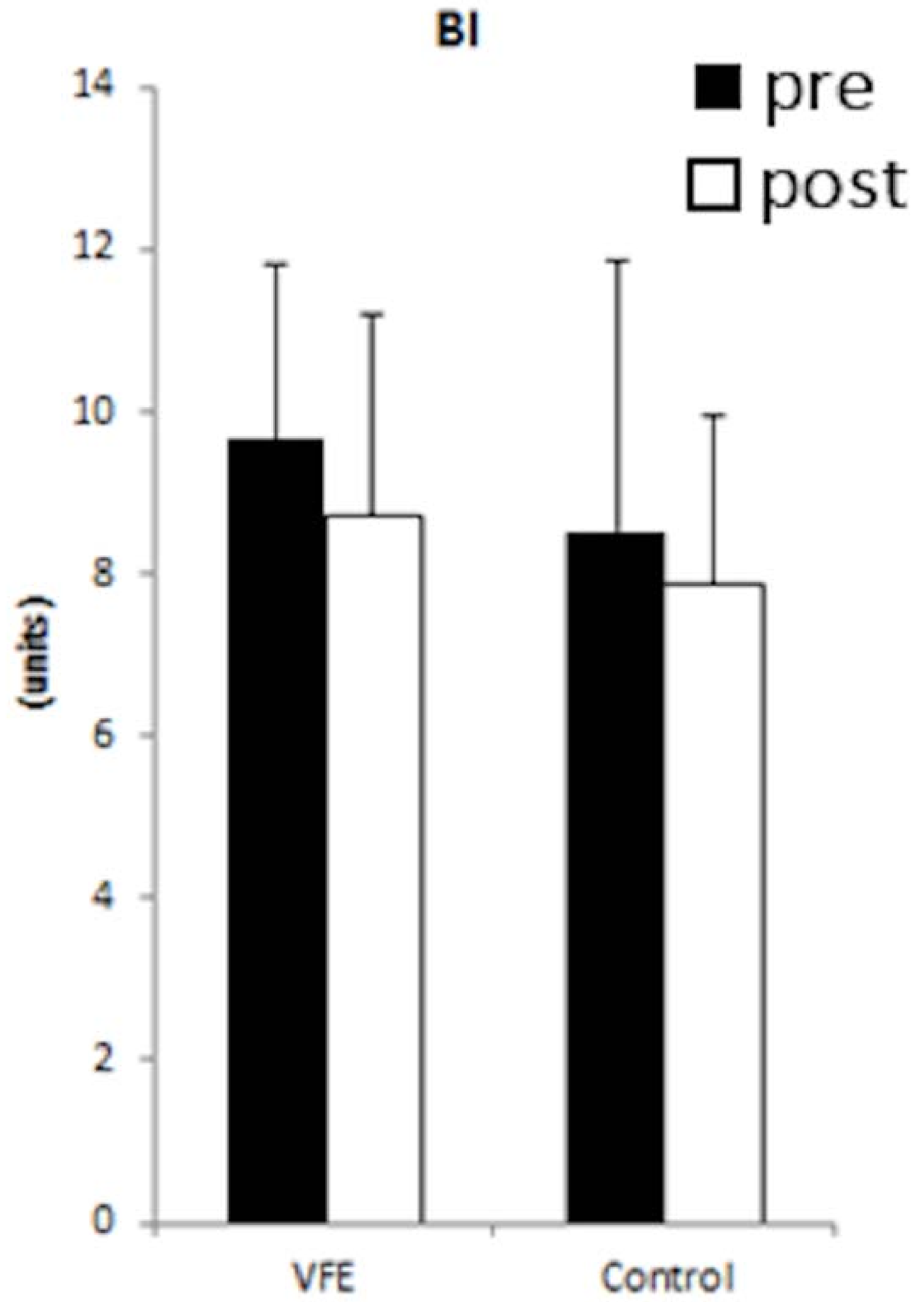

